\title{
Retos en la incorporación al mercado laboral: análisis de la brecha en competencias Challenges in entering the labour market: a competence gap analysis
}

\author{
M.C. Riera Prunera ${ }^{1}$, Y. Blasco Martel ${ }^{2}$, L. Duque ${ }^{3}$, J. López Tamayo ${ }^{1}$, M. Pujol Jover ${ }^{4}$ \\ mcriera-prunera@ub.edu, yolandablasco@ub.edu, lduque@emp.uc3m.es, jlt_lopez@ub.edu, mpujoljo@uoc.edu \\ ${ }^{1}$ Dep. de Econometría, \\ Estadística y E. Aplicada \\ Universitat de Barcelona \\ Barcelona, Spain \\ ${ }^{2}$ Dep. Historia e \\ Instituciones Económicas \\ Universitat de Barcelona \\ Barcelona, Spain \\ ${ }^{3}$ Dep. de Economía y \\ Empresa \\ Universidad Carlos III \\ Madrid, Spain \\ ${ }^{4}$ Dep. de Economía y \\ Empresa \\ Universitat Oberta (UOC) \\ Barcelona, Spain
}

\begin{abstract}
Resumen- Este artículo analiza el nivel de adquisición de competencias básicas y transversales a través de la autoevaluación realizada por los alumnos y la valoración realizada por las empresas en las que los alumnos han realizado prácticas durante las etapas finales de sus estudios. Para ello preparamos y distribuimos dos cuestionarios, uno para los estudiantes que desarrollaron las prácticas y otro para los tutores de estudiantes de las empresas. Para analizar los resultados nos hemos centrado en los procedimientos asociados con el análisis de varianza, ANOVA. Más concretamente hemos aplicado el modelo SERVQUAL. Este modelo considera la presencia de diferencias en términos de expectativas y percepciones entre las dos partes implicadas en una prestación de servicios. Identificar las principales brechas en el desarrollo y la evaluación de las competencias de los estudiantes se convierte en un tema clave, ya que es crucial a la hora de determinar no sólo la transición al mercado de trabajo, sino cómo alcanzar las habilidades que los estudiantes deben tener al inicio de su carrera profesional y, por lo tanto, sus posibilidades de éxito o fracaso, las probabilidades de un buen desempeño en la empresa o su capacidad de aprendizaje.
\end{abstract}

Palabras clave: análisis de discrepancias, competencias instrumentales, competencias interpersonales, competencias profesionales, test ANOVA.

Abstract- This article studies the acquisition level of basic and transversal skills through a self assessment made by the students and the assessment made by the companies where students have carried out internships during the final stages of their studies. To do this we prepared and distributed two questionnaires, one for the students who completed internships in a firm and another one for the firms' students' tutors. To analyse the results we have focused on procedures associated with the analysis of variance, ANOVA. More specifically we have applied the SERVQUAL model. This model considers the presence of differences in terms of expectations and perceptions between the two parties involved in a provision of services. Identifying the main gaps in the development and assessment of the students' skills becomes a key issue, since it is crucial when determining not only their transition to the labour market, but how to achieve the skills level students must have when they start working and, therefore, their chances of success or failure, the odds of a good performance in the company, or their learning ability.

Keywords: gap analysis, instrumental competences, interpersonal competences, professional competences, ANOVA test.

\section{INTRODUCCIÓN}

En el entorno actual, donde la sociedad exige capacidad visión, emprendimiento y generación de nuevas ideas, la formación de los graduados universitarios es crucial. El nuevo modelo de aprendizaje basado en la adquisición de competencias requiere de herramientas de aprendizaje modernas que permitan a los graduados desarrollar habilidades $\mathrm{y}$ convertirse en constructores activos de conocimiento en lugar de ser receptores pasivos de contenidos. En este sentido, Serradell-López et al. (2013) y Munroe y Westwind (2009) destacan el papel crítico de la universidad como centro de conocimiento e innovación, destacando su profunda importancia en la construcción de una economía fuerte. Mientras tanto, Etzkowitz (2003) hace hincapié en la necesidad de enriquecer la interacción entre la universidad, las empresas y el gobierno.

Por esta razón, la adquisición de un alto grado de competencias es sumamente importante, ya que facilita la transición de los graduados al mercado de trabajo. Salas Velasco (2014), Chillas, 2010, Boccuzzo y Gianecchini (2015), refuerzan esta idea, destacando que la velocidad con que los graduados aprenden a desarrollar su trabajo se basa en el nivel y tipo de habilidades adquiridas. Moore, y Murphy (2009) añade que los graduados, que aprenden a gestionar su propio aprendizaje durante su tiempo en la universidad, podrán gestionar mejor sus necesidades profesionales con iniciativa, creatividad y un elevado grado de autonomía.

Desde el punto de vista empresarial, existen numerosos estudios que muestran ésta necesidad, tanto a nivel nacional (Marzo Navarro et al., 2008, Alcañiz-Zanón, et al., 2014, Alcañiz, et al., 2013) como a nivel internacional (Biesma, et al. 2007, Bridgstock, 2009, Crossman y Clarke, 2010, Branine y Avramenko, 2015). A partir de la evaluación del grado de satisfacción de los empleadores con los graduados, se observa que existe una gran discrepancia y en consecuencia muchas cosas que corregir. Esto no se debe generalmente a la falta de conocimientos específicos de la profesión por parte de los graduados, sino a la falta de competencias, que a veces resulta ser una barrera insalvable al aplicar lo que han aprendido a un contexto profesional. AcNielsen Research Services (2000) en 
el informe para el gobierno australiano, hace hincapié en esta idea llegando a la conclusión de que los graduados, que son rechazados por la empresa, tienden a ser los que tienen menores niveles competenciales. De hecho, las empresas a menudo prefieren contratar graduados en lugar de trabajadores menos calificados, sólo porque se supone que tienen mejores habilidades.

En este contexto, este trabajo pretende mostrar que el acercamiento entre el mundo académico y el mundo empresarial tiene todavía un largo camino por recorrer, dado que se observan fuertes diferencias conceptuales y prácticas entre lo que la universidad enseña a sus graduados y lo que las empresas buscan y aprecian de los nuevos graduados que empiezan trabajar para ellos. Sobre la base de un estudio de caso, en el presente trabajo se analizan cuáles son las principales discrepancias entre las habilidades formativas que requieren las empresas de los graduados y las habilidades y conocimientos adquiridos por los mismos antes de entrar en el mercado de trabajo.

El análisis realizado en este trabajo se basa en medidas subjetivas (percepciones); un enfoque que se utiliza cada vez más en la literatura de educación superior (González y Wagenaar, 2003, Rasli et al., 2012, Duque y Weeks, 2010). Específicamente, profundizamos en las cuatro posibles brechas existentes entre los dos grupos que se derivan del modelo SERVQUAL propuesto por Parasuraman et al. (1985).

El resto del documento está organizado de la siguiente manera. A continuación, se explica el marco de análisis y metodología aplicada; Entonces presentamos nuestro caso de análisis detallando los principales hallazgos y discutiendo los resultados. El documento finaliza con las conclusiones extraídas del análisis.

\section{CONTEXTO}

En el origen del proceso de creación de un Espacio Europeo de Educación Superior (EEES) se encontraba el proyecto Tuning, cuyo objetivo era "sintonizar" o sincronizar las realidades universitarias europeas. Así, el EEES es el ámbito en el que las universidades europeas han acordado la estrategia de convergencia que permite la homologación de títulos de educación superior en el territorio europeo. El proceso de convergencia en la educación superior, también conocido como Proceso de Bolonia, fue uno de los objetivos fijados por la Comisión Europea en Lisboa en 2000 (la Estrategia de Lisboa). Los títulos del sistema universitario europeo, en el marco del EEES, se basan en el Sistema Europeo de Transferencia y Acumulación de Créditos (ECTS). Los créditos ECTS favorecen la armonización de los diplomas europeos, así como la movilidad de estudiantes y profesionales en todo el territorio europeo.

Para poder comparar los grados era necesario establecer un nivel mínimo de competencias evaluadas al final del grado que todos los estudiantes deberían alcanzar. Las competencias fueron definidas como:

"(...) una combinación dinámica de capacidades cognitivas y metacognitivas, conocimiento y comprensión, valores interpersonales, intelectuales y prácticos, así como valores éticos, fomentar estas competencias es el objetivo principal de todos los sistemas educativos (...). Las competencias se desarrollan en todas las unidades o partes del temario y se evalúan en diferentes partes del curso. Éstas se pueden dividir en competencias relacionadas con disciplinas (específicas de un campo de estudio) y competencias genéricas (comunes a cualquier grado) ${ }^{1}$.

A partir de este marco, se definieron y recopilaron las competencias que caracterizan los distintos grados en las distintas fases del proyecto Tuning (González y Wagenaar, 2003, 2006). En este punto, cada contexto universitario nacional definió, de acuerdo con sus gobiernos, su forma particular de incorporarse al EEES de manera que las universidades pudiesen mantener cierto grado de autonomía para mantener su identidad. Así, la priorización en las competencias a desarrollar tanto como el grado de aceptación de sus graduados al ingresar al mercado de trabajo definirían el sello de identidad de la universidad.

El Parlamento Europeo ha supervisado la aplicación del proceso de Bolonia, emitiendo una resolución el 28 de abril de 2015 (2015/2039 (INI)), en la que se señala la necesidad de evaluar los progresos ya realizados, invitando a todas las partes a profundizar en su mejora (básicamente con respecto a la garantía de calidad) involucrando tanto a gobiernos como a universidades. Asimismo, también indica que:

"El análisis realizado hasta la fecha demuestra que casi uno de cada tres empleadores de la UE tiene dificultades para localizar empleados cualificados, por lo que la reforma de Bolonia no ha tenido mucho éxito en la reducción de la insuficiencia de cualificaciones en la UE. La brecha entre las cualificaciones profesionales y la demanda del mercado de trabajo sigue siendo elevada. Este desajuste se ha convertido en un desafío crucial para Europa, que abarca diferentes esferas de la sociedad, desde la productividad y la eficiencia de las empresas hasta el bienestar actual y futuro de los jóvenes. (...) Aunque el proceso de Bolonia ha guiado $\mathrm{y}$ motivado las reformas educativas en la mayoría de los países, en otros puede ser percibido como una carga burocrática debido a la mala comunicación y la falta de comprensión de su verdadera visión".

En esta línea, uno de los desafíos actuales del EEES que está fuertemente asociado con nuestro trabajo es promover la empleabilidad de los titulados universitarios (Ministros europeos responsables de la educación superior, 2009, 2-5).

\section{DESCRIPCIÓN}

El modelo SERVQUAL (SERVice QUALity) está basado en cinco discrepancias o gaps, el más destacado de los cuales es la diferencia que hay entre lo que los clientes esperaban y lo que finalmente percibieron con el servicio. Esta diferencia principal se produce como consecuencia de los cuatro gaps restantes: carencia de conocimiento de las expectativas del otro, especificaciones del servicio tal y como son entendidas por los empleados, maneras diferentes de comunicación que conforman las expectativas de los clientes, y los recursos disponibles para entregar el servicio. Creemos que es posible adaptar este modelo a la comparativa entre las percepciones que tienen los tutores y los estudiantes respecto a las competencias requeridas y adquiridas, siguiendo lo hecho por algunos autores, cuyos estudios cubren distintos aspectos de la educación superior (Zafiropoulos y Vrana, 2008), tales como comparar percepciones y expectativas entre estudiantes $\mathrm{y}$

\footnotetext{
${ }^{1}$ Parasuraman et al., 1985, p.3.
} 
académicos, analizar las percepciones según tengan lugar en niveles diferentes de una institución, comparar perspectivas de empresarios con las de otros agentes sociales, desarrollar medidas específicas para programas de posgrado, etc. Además, Firdaus (2006) identifica seis dimensiones relacionadas con la calidad del servicio, que son relevantes en lo que a la educación superior respecta.

En este estudio aplicamos el modelo SERVQUAL para analizar las perspectivas de dos de los actores principales en la prestación de educación superior: estudiantes (clientes directos del servicio) y tutores (beneficiarios indirectos por el lado del mercado del trabajo). Uno de los aspectos claves de nuestra aproximación es que no entramos en las dimensiones de calidad del servicio, sino que lo hacemos a nivel de las competencias desarrolladas en la Universidad. Nuestro marco de análisis considera 4 gaps. El Gap A se enfoca hacia las competencias que los tutores observan en los estudiantes en prácticas, comparándolas con las que esperan encontrar. El Gap B captura lo que los estudiantes piensan es el nivel de competencias que han adquirido durante sus estudios, comparándolas con las que piensan les están siendo requeridas por las empresas. El Gap C tiene en cuenta ambas perspectivas y lleva a cabo el análisis en términos de expectativas (competencias requeridas en el trabajo). Finalmente, el Gap D se focaliza en la dualidad del análisis en términos del nivel de competencias mostrado en trabajo. Esta última discrepancia es bastante reveladora dado que muestra la valoración directa de la percepción de las competencias que se produce. La Figura 1 muestra el modelo conceptual que se ha utilizado para este análisis dual.

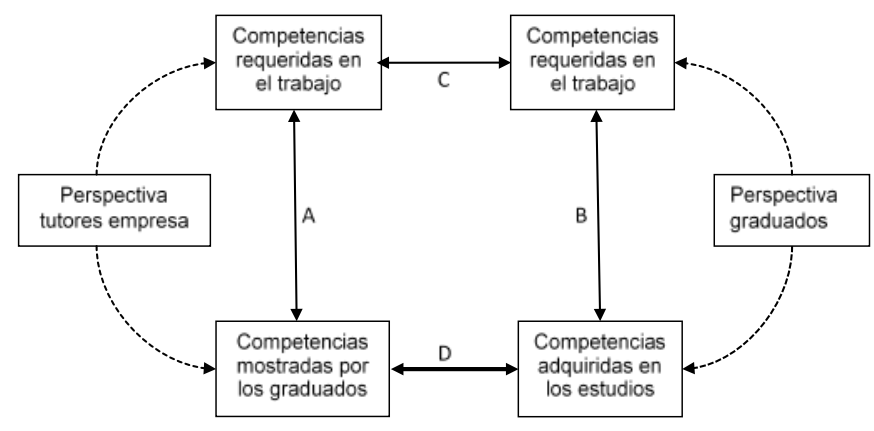

Figura 1. Marco para análisis de discrepancias.

Fuente: elaboración propia

Para llevar a cabo nuestro análisis elaboramos y distribuimos dos cuestionarios: uno para los estudiantes de la Universitat de Barcelona que habían hecho las prácticas en una empresa bajo el programa de las "Becas Santander”, y otro para los tutores que las empresas asignaron a cada uno de los estudiantes, asegurándonos que los ambos conjuntos de competencias fueran directamente comparables.

Analizamos las discrepancias correspondientes a 38 competencias agrupadas en tres conjuntos: instrumentales (11), interpersonales (12) y profesionales (15). De los 192 tutores y estudiantes, 151 tutores y 81 estudiantes respondieron el cuestionario. Bajo la suposición de máxima indeterminación $(\mathrm{p}=\mathrm{q}=0.5)$, y con un $95 \%$ de nivel de confianza, el error muestral es de un $3.69 \%$ y el error de estimación del 8.3\%. Dado que ambos cuestionarios eran anónimos creemos que no existe ningún otro sesgo excepto el de no-respuesta, tal y como es habitual en la mayoría de encuestas (p. ej. Lefever et al., 2007).

El cuestionario evaluó cada competencia a través de una escala cuantitativa de Likert de 1 a 10, donde 10 es la valoración más elevada. A los tutores se les preguntó que valoraran el nivel de cada competencia requerido para el trabajo, así como el nivel mostrado por los estudiantes en las tareas que desempeñaban. Los estudiantes, a su vez, fueron preguntados por el nivel que de cada competencia se les requirió en la empresa, así como por el nivel en que habían adquirido tales competencias durante sus estudios.

El análisis de discrepancias fue llevado a cabo utilizando el programa estadístico SPSS-23. Utilizamos el análisis de la variancia ANOVA para evaluar las discrepancias existentes entre tutores y estudiantes, y también para capturar la opinión de cada grupo con respeto al rendimiento versus las expectativas (el nivel de significación era del 0.05 y las varianzas entre los dos grupos se supusieron distintas). En línea con las recomendaciones de literatura sobre marketing (Rossiter, 2002) comprobamos la consistencia de los tres conjuntos de competencias (Cronbach, 1951) para crear los índices globales que permiten llevar a cabo una comparación general en términos del Gap D.

\section{Resultados}

Nuestros resultados hacen referencia a las cuatro categorías que se desprenden de las discrepancias analizadas (ver Tabla1). Para cada conjunto de competencias detallamos las discrepancias más relevantes, basándonos en el tamaño de las diferencias estadísticamente significativas.

Las columnas 5 y 6 en la Tabla 1 muestran las divergencias entre lo que los tutores perciben y el nivel competencial requerido para el trabajo (Gap A). Por lo que respecta a las competencias instrumentales y las interpersonales, aproximadamente la mitad de las discrepancias resultan ser significativas, pero esta cifra sube a los dos tercios cuándo analizamos las competencias profesionales.

Todas las discrepancias significativas resultan ser negativas. Esto significa que desde la perspectiva de los tutores a los estudiantes les queda aún un cierto camino por recorrer para para mejorar sus logros competenciales. Con respecto a las competencias instrumentales e interpersonales, necesitan mejorar en los siguientes aspectos concretos: análisis y síntesis, organización y planificación, conocimiento aplicado a la práctica, solución de problemas, trabajo autónomo, comunicación oral en la propia lengua, habilidades críticas, trabajo en equipo y trabajo debajo presión. Con respeto a las competencias profesionales, los tutores consideran que los estudiantes han conseguido eficazmente el nivel requerido en términos de vocabulario económico, habilidad para redactar informes técnicos, conocimientos macro del entorno, estrategias de internacionalización e implicaciones de políticas públicas, mientras que en el resto, tendrían que mejorar su nivel.

Vale la pena remarcar que hay una única competencia que los tutores percibieron como "sobre-conseguida" por los estudiantes, concretamente "impacto medioambiental”.

Las columnas 7 y 8 de la Tabla 1 muestran las diferencias percibidas por los estudiantes con respecto a lo que piensan que es el nivel estándar competencial conseguido en la 
universidad y el nivel realmente requerido en la empresa (Gap B). Así, los estudiantes perciben la existencia de discrepancias en 10 de las 38 competencias analizadas. En 7 de ellas consideran que no han conseguido el nivel requerido en la empresa: habilidades informáticas aplicación de conocimiento a la práctica, capacidad para adaptarse a situaciones nuevas, iniciativa y emprendeduría, conocimiento interno de la empresa, conocimiento de estrategias internacionales e implicaciones de las políticas gubernamentales. Como puede verse, corresponden básicamente al grupo de competencias profesionales. En contraste con esto, los estudiantes creen que están sobrecualificados en términos de conocimientos generales y específicos, así como apreciación de la multiculturalidad.

Las columnas 9 y 10 de la Tabla 1 ilustran las discrepancias entre estudiantes y los tutores con respecto a las habilidades requeridas (Gap C). En este caso, sólo en 3 de los 38 casos existen diferencias estadísticamente significativas. Los estudiantes consideran que se les requiere un nivel más alto del que en realidad los tutores hacen en términos de responsabilidad y toma de decisiones. Todo lo contrario de lo que sucede en relación a los conocimientos específicos y de una lengua extranjera.

Finalmente, las columnas 11 y 12 de la Tabla 1, presentan las disparidades entre lo que estudiantes perciben como conseguido en la universidad y lo que los tutores observan (Gap D). En este aspecto, los estudiantes parecen infravalorar su nivel competencial en lo que respecta a conocimiento de una lengua extranjera, aplicación de conocimiento a la práctica, ética empresarial, conocimiento interno de la empresa e implicaciones de las políticas gubernamentales. Contrariamente, sobrevaloran su logro en la habilidad de trabajar autónomamente y en la conclusión e interpretación de resultados. En este caso es interesante comprobar que todas las discrepancias significativas se concentran en las habilidades instrumentales y profesionales.

\section{CONCLUSIONES}

Este papel analiza la relación entre las competencias desarrolladas en la universidad y aquellas que demanda el mercado laboral basándose en un modelo de discrepancias. Para detectar las diferencias significativas en la percepción de cuáles son las habilidades más importantes para un buen desarrollo de la carrera profesional de un estudiante de grado, se observa el grupo en el que realizan la evaluación los estudiantes y los tutores de empresa.

Las principales conclusiones que arroja el análisis de discrepancias es que (i) los estudiantes no han adquirido todo el conocimiento específico que aparentemente sería útil en su futuro inmediato para incorporarse de modo exitoso en el mercado laboral (Gap A); (ii) los estudiantes parecen carecer de cierto grado de autoestima y autoconfianza en sus habilidades y conocimientos (Gap B, donde se concentran las divergencias negativas más importantes). Esto está alineado con Conchado et al. (2015) y Brachem y Braun (2015). Sin embargo, una conclusión alentadora es que las percepciones, tanto de los empleadores como de los estudiantes sobre las competencias requeridas, parecen funcionar en la misma dirección (Gap C). Además, con relación a lo que se les exige en el trabajo, la evaluación de ambas partes se aproxima bastante, lo que implica las menores disparidades en el análisis.

Del trabajo se concluye que la universidad tendría que concentrar sus esfuerzos, especialmente, en incrementar las capacidades cuyas diferencias están presentes en la mayoría de los gaps. Para ello sería necesario un contacto más directo y más profundo entre la universidad y las empresas. En este sentido, las empresas deberían desempeñar, junto con las universidades, un papel activo en el diseño de algunas estrategias, metodologías y contenidos universitarios. Por ejemplo, podrían organizarse congresos, seminarios y conferencias conjuntas enfocadas para alcanzar los requisitos exigidos por las asignaturas. Este tipo de actividades debería centrar su atención, especialmente, en fomentar el trabajo en equipo y la comunicación. Además, debería introducirse en las primeras etapas de los grados el contacto directo con las empresas a través de pasantías y colaboraciones específicas con objeto de facilitar la necesaria interacción entre los estudiantes y las empresas. También sería importante que los proyectos finales de los estudiantes se desarrollaran en una empresa específica. En esta línea cabe destacar el valioso avance que ha supuesto a la hora de profundizar en el contacto universidad-empresa el programa de prácticas en empresas internacionales asociado al proyecto europeo VALS que lleva a cabo la Universidad de Salamanca (García-Peñalvo et al., 2015).

En resumen, es importante que tanto los empleadores como la universidad actúen reforzando la autoconfianza de los estudiantes lo que sin duda les ayudará en términos de mejor desempeño al ingresar en el mercado de trabajo. No obstante, deberíamos que preguntarnos primero qué tipo de mercado laboral queremos tener, qué economía nos dirigirá nuevamente a un camino de crecimiento estable y, en base a esto, decidir qué tipo de educación universitaria deberían recibir los estudiantes. La colaboración entre el mundo empresarial y el académico es deseable con objeto de que nuestros graduados reciban la mejor formación posible, lo que, sin duda, colaboraría a la mejora de la productividad de nuestra economía. Es imprescindible que el currículo universitario preparare graduados para su ingreso el mercado laboral proporcionándoles conocimiento, habilidades, y comportamientos que no sólo necesitan las empresas sino también la sociedad. Por otra parte, también cabe exigir a los empresarios que piensen en el mejor perfil de los empleados, intentando que sus exigencias y demandas sean más explícitas y que las hagan con una visión de largo plazo. Queda por responder la cuestión de si la búsqueda de la inmediatez y la eficacia pudieran comportar la renuncia al conocimiento más estricto y al avance intelectual en el futuro.

\section{AGRADECIMIENTOS}

Los autores agradecen la ayuda recibida a través del proyecto REDICE16-1562 Labour and training needs and requirements for graduates. Analysing the existing competence gap on the basis of firm internships, financiado por el Institut de Ciències de l'Educació (ICE), Universitat de Barcelona, 2016. También a la Borsa de treball de la Universidad de Barcelona por su colaboración en el diseño de los cuestionarios y especialmente en su distribución a las distintas facultades. 


\section{REFERENCIAS}

AcNielsen Research Services. 2000. Employer Satisfaction with Graduate Skills. Research Report. http://www.voced.edu.au/content/ngv13863 Recuperado el 3 de Noviembre de 2015.

Alcañiz, M., Riera, C., y Claveria, O. (2013). La formación competencial de los licenciados en Economía y Empresa: una visión desde su entorno profesional. Revista d'Innovació i Recerca en Educació, 6(2), 64-85.

Alcañiz-Zanón, M., Claveria-González. O., y Riera-Prunera, M.C. (2014). Competencias en educación superior desde tres perspectivas diferentes: estudiantes, empleadores y académicos. Revista Iberoamericana de Educación (RIE), 66(2), 1-19.

Biesma, R.G., Pavlova, M., Van Merode, G.G., y Groot, W. (2007). Using conjoint analysis to estimate employers preferences for key competencies of master level Dutch graduates entering the public health field. Economics of Education Review, 26(3), 375-386.

Boccuzzo, G., y Gianecchini, M. (2015). Measuring young graduates' job quality through a composite indicator. Social Indicators Research, 122, 453-478.

Brachem, J., y Braun, E. M. P. (2015). Requirements higher education graduates meet on the labor market. Peabody Journal of Education, 90(4), 574-595.

Branine, M., y Avramenko, A. (2015). A comparative analysis of graduate employment prospects in european labour markets: A study of graduate recruitment in four countries. Higher Education Quarterly, 69(4), 342-365.

Bridgstock, R. (2009). The graduate attributes we've overlooked: Enhancing graduate employability through career management skills. Higher Education Research y Development, 28(1), 31-44.

Chillas, S. (2010). Degrees of fit? Matching in the graduate labour market. Employee Relations, 32(2), 156-170.

Conchado, A., Carot, J. M., y Bas, M. C. (2015). Competencies for knowledge management: Development and validation of a scale. Journal of Knowledge Management, 19(4), 836-855.

Cronbach, L. J. (1951). Coefficient alpha and the internal structure of test. Psichometrica, 16, 297-334.

Crossman, J. E., y Clarke, M. (2010). International experience and graduate employability: Stakeholder perceptions on the connection. Higher Education, 59(5), 599-613.

Duque, L.C., y Weeks, J.R. (2010). Towards a model and methodology for assessing student learning outcomes and satisfaction. Quality Assurance in Education, 18(2), 84-105.

Etzkowitz, H. (2003). Innovation in Innovation: the Triple Helix of University-Industry-Government Relations. Social Science Information sur les Sciences Sociales, 42(3), 293-337.

Firdaus, A. (2006). The development of HEdPERF: a new measuring instrument of service quality for higher education, International Journal of Consumer Studies, 30(6), 569-581.
García-Peñalvo, F. J., Cruz-Benito, J., Griffiths, D., y Achilleos, A. (2015). Tecnología al servicio de un proceso de gestión de prácticas virtuales en empresas: Propuesta y primeros resultados del Semester of Code. VAEP RITA.

González, J. y Wagenaar, R. (Ed). (2003). Tuning Educational Structures in Europe. Informe Final Fase I. Bilbao, España: Universidad de Deusto.

González, J. y Wagenaar, R. (Ed). (2006). Tuning Educational Structures in Europe II. La contribución de las universidades al proceso de Bolonia. Bilbao, España: Universidad de Deusto.

Lefever, S., Dal, M., y Matthíasdóttir, A. (2007). Online data collection in academic research: advantages and limitations. British Journal of Educational Technology, 38, 574-582.

Marzo Navarro, M., Pedraja Iglesias, M., y Rivera Torres, P. (2008). Determinants of the Satisfaction of Firms with the Competencies of University Students: a Spanish Case Study. International Journal of Training and Development, 12(4), 282-292.

Moore, S., y Murphy, M. (2009). Estudiantes Excelentes. 100 Ideas Prácticas para Mejorar el Autoaprendizaje en Educación Superior. Madrid: Narcea.

Munroe, T. y Westwind, M. (2009). What Makes Silicon Valley Tick? The Ecology of Innovation at Work. New York: Nova Vista Publishing.

Parasuraman, A., Zeithaml, V.Z., y Berry, L. (1985). A Conceptual Model of Service Quality and its Implications for Future Research. Journal of Marketing, 49(3), 41-50

Rasli, A., Shekarchizadeh, A., y Jawad Iqbal, M. (2012). Perception of Service Quality in Higher Education: Perspective of Iranian Students of Malaysian Universities. Int. Journal of Economics and Management 6(2), $201-220$.

Rossiter, J. R. (2002). The C-OAR-SE procedure for scale development in marketing. International Journal of Research in Marketing, 19, 305-335.

Salas Velasco, M. (2014). Do higher education institutions make a difference in competence development? A model of competence production at university. Higher Education, 68(4), 503-523.

Serradell-López, E., Fitó Bertran, A., Pujol-Jover, M., Dalton, V., Hempsall, K., y Hernandez-Lara, A. B. (2013). Online Tools for Management Skills Development. Poster presented at the First UOC International Research Symposium, Barcelona, December 18th.

Tazreen, S. (2012). An Empirical Study of Servqual as a Tool for Service Quality Measurement. IOSR Journal of Business and Management, 1(5), 9-19.

Zafiropoulos, C., y Vrana, V. (2008). Service quality assessment in a Greek higher education institute. Journal of Business Economics and Management, 9(1), 33-45. 
Tabla 1: Valoración de las percepciones de los tutores y estudiantes en prácticas. Análisis de discrepancias.

\begin{tabular}{|c|c|c|c|c|c|c|c|c|c|c|c|c|}
\hline \multirow{2}{*}{ Competencias $^{\mathrm{a}}$} & \multicolumn{2}{|c|}{ Tutores } & \multicolumn{2}{|c|}{ Estudiantes } & \multicolumn{2}{|c|}{ GAP A } & \multicolumn{2}{|c|}{ GAP B } & \multicolumn{2}{|c|}{ GAP C } & \multicolumn{2}{|c|}{ GAP D } \\
\hline & Req. & Obs. & Req. & Cons. & Diff. & ANOVA & Diff. & ANOVA & Diff. & ANOVA & Diff. & ANOVA \\
\hline Instrumentales & $(1)$ & $(2)$ & (3) & (4) & (5) & (6) & $(7)$ & $(8)$ & (9) & $(10)$ & $(11)$ & $(12)$ \\
\hline 1 Análisis y síntesis & 7,88 & 8,07 & 8,31 & 8,51 &,- 185 & $041^{* *}$ & , 198 & 328 & 185 &, 514 & ,370 & 111 \\
\hline 2 Organización y planificación & 7,90 & 8,13 & 8,41 & 8,48 &,- 227 &, $023 * *$ & 074 & ,752 & 238 & 423 & ,395 & 134 \\
\hline 3 Conocimiento básico general & 7,98 & 8,03 & 7,96 & 8,64 &,- 057 & 497 & 679 &, $003 * * *$ &,- 333 & ,227 & ,350 & 148 \\
\hline 4 Conocimiento específico del grado & 7,95 & 8,04 & 7,32 & 8,15 &,- 082 &, 330 &, 827 &, $003 * * *$ & $-1,062$ &, $003 * * *$ &,- 160 & 469 \\
\hline 5 Conocimiento de una lengua extranjera & 7,70 & 7,54 & 7,04 & 6,81 & ,155 & 160 &,- 222 & ,425 &,- 802 &, $033^{* * *}$ & $-1,123$ &, $001 * * *$ \\
\hline 6 Habilidades informáticas & 7,85 & 7,98 & 8,32 & 7,46 &,- 130 & 149 &,- 864 &, $001 * * *$ & 0,000 & 1,000 &,- 463 &, 120 \\
\hline 7 Aplicación del conocimiento a la práctica & 7,95 & 8,28 & 8,20 & 7,59 &,- 330 &, $001 * * *$ &,- 605 &, $024 * *$ &,- 259 & ,407 &,- 617 & ,031** \\
\hline 8 Solución de problemas & 7,83 & 8,12 & 8,49 & 8,06 &,- 285 &, $004 * * *$ &,- 432 &, 143 &, 222 & 452 &,- 111 & 684 \\
\hline 9 Organización de la información & 8,16 & 8,27 & 8,63 & 8,53 &,- 098 & 235 &,- 088 & 696 & ,222 & 450 & ,138 & 613 \\
\hline 10 Trabajo autónomo & 7,91 & 8,33 & 8,66 & 8,78 &,- 409 &, $000 * * *$ & 125 & 601 & 288 & 261 &, 700 &, $007 * * *$ \\
\hline 11 Toma de decisiones & 7,70 & 7,85 & 8,19 & 8,35 &,- 186 & ,076* & ,139 & 617 & 238 & ,413 & ,375 & 147 \\
\hline \multicolumn{13}{|l|}{ Interpersonales } \\
\hline 1 Comunicación oral en catalán/castellano & 8,38 & 8,53 & 8,58 & 8,74 &,- 152 & ,046** & ,256 & 234 & 132 & 687 & 367 & ,172 \\
\hline 2 Comunicación escrita en catalán/castellano & 8,29 & 8,39 & 8,71 & 8,74 &,- 137 & $092 *$ & 128 & 552 & 382 & ,266 & ,494 & $070 *$ \\
\hline 3 Habilidad de crítica y auto-crítica & 7,93 & 8,10 & 8,14 & 8,35 &,- 189 & $034 * *$ & ,205 & 406 & 120 & 724 & ,380 & ,189 \\
\hline 4 Trabajo en equipo & 8,16 & 8,48 & 8,35 & 8,11 &,- 304 &, $001^{* * *}$ &,- 231 & 414 &,- 118 & ,707 &,- 038 & 893 \\
\hline 5 Liderazgo & 7,08 & 7,11 & 6,94 & 7,18 &,- 011 & ,924 & ,218 & ,476 &,- 280 & ,447 &,- 167 & 626 \\
\hline 6 Habilidad para trabajar bajo presión & 7,51 & 7,74 & 8,23 & 8,16 &,- 211 & $046^{* *}$ &,- 090 & ,744 & ,627 & ,089* &, 557 & $069 *$ \\
\hline 7 Habilidad para transmitir conocimiento & 7,46 & 7,61 & 7,71 & 8,18 &,- 132 & 184 &, 500 &, $092 *$ & 0,000 & 1,000 &, 551 &, $065 *$ \\
\hline 8 Habilidades negociadoras & 7,11 & 7,23 & 7,20 & 7,18 &,- 075 & 460 & 0,000 & 1,000 &,- 378 & ,292 &,- 221 &, 544 \\
\hline 9 Apreciación del multiculturalismo & 7,94 & 7,71 & 7,29 & 8,01 &, 176 &, $051 *$ & ,831 &, $005 * * *$ &,- 200 & 627 &,- 013 & ,967 \\
\hline 10 Habilidad para imponer autoridad & 6,98 & 6,89 & 6,63 & 6,65 & ,108 & ,310 & 077 & ,799 &,- 211 & ,603 &,- 418 & ,266 \\
\hline 11 Diseño y dirección de proyectos & 7,45 & 7,51 & 7,68 & 7,34 &,- 048 & ,661 &,- 286 & ,317 &,- 120 & ,751 &,- 342 & ,331 \\
\hline 12 Impacto medioambiental y social & 7,48 & 7,14 & 6,92 & 7,03 & ,324 &, $008 * * *$ &, 160 &, 636 &,- 178 & ,698 &,- 474 &, 231 \\
\hline \multicolumn{13}{|l|}{ Profesionales / Sistémicas } \\
\hline 1 Habilidad para adaptarse a situaciones nuevas & 7,97 & 8,16 & 8,68 & 7,92 &,- 201 &, $020 * *$ &,- 757 &, $002 * * *$ & ,581 & ,071* &,- 096 & ,702 \\
\hline 2 Capacidad para aprender & 8,26 & 8,48 & 8,73 & 8,62 &,- 222 &, $013 * *$ &,- 110 & ,637 & ,301 & ,343 &, 247 & ,337 \\
\hline 3 Creatividad & 7,74 & 8,07 & 8,05 & 7,54 &,- 340 &, $001 * * *$ &,- 500 & ,057* & 027 & 937 &,- 260 & ,423 \\
\hline 4 Iniciativa y emprendiduría & 7,71 & 8,20 & 8,07 & 7,36 &,- 497 &, $000 * * *$ &,- 671 &, $008 * * *$ &,- 055 & 873 &,- 301 & ,345 \\
\hline 5 Auto-exigencia y preocupación por el éxito & 7,81 & 8,28 & 8,45 & 8,26 &,- 470 &, $000 * * *$ &,- 189 & ,449 & ,274 & ,434 & ,384 & 209 \\
\hline 6 Responsabilidad en la toma de decisiones & 7,62 & 7,99 & 8,51 & 8,07 &,- 385 &, $001 * * *$ &,- 384 &, $092 *$ & 616 &, $043^{* *}$ & ,356 & 249 \\
\hline 7 Uso de vocabulario económico & 7,04 & 7,17 & 7,00 & 6,95 &,- 152 & 173 &,- 054 & 870 & 0,000 & 1,000 &,- 153 & 678 \\
\hline 8 Conclusión e interpretación de resultados & 7,58 & 7,98 & 8,25 & 8,26 &,- 441 &, $000 * * *$ &,- 014 & ,955 & ,311 &, 319 &, 753 &, $011 * *$ \\
\hline 9 Habilidad para hacer informes técnicos & 7,43 & 7,56 & 8,05 & 7,65 &,- 167 & 145 &,- 370 & 142 & ,403 & ,286 & ,192 & ,513 \\
\hline 10 Ética empresarial & 7,56 & 7,91 & 7,27 & 6,72 &,- 333 & $003 * * *$ &,- 569 & ,058* &,- 608 & 102 &,- 831 &, $023^{* *}$ \\
\hline 11 Visión global & 7,40 & 7,75 & 7,73 & 7,69 &,- 379 &, $000 * * *$ &,- 054 & ,853 & ,027 & 937 & ,333 & 294 \\
\hline 12 Conocimiento interno de la empresa & 7,17 & 7,35 & 7,49 & 6,41 &,- 219 & $034 * *$ & $-1,081$ &, $001 * * *$ & 162 & 648 &,- 726 &, $045 * *$ \\
\hline 13 Conocimiento del entorno Macro & 7,04 & 7,05 & 6,95 & 6,49 &,- 066 &, 544 &,- 446 & 118 &,- 189 & 621 &,- 542 & 145 \\
\hline 14 Estrategias de internacionalización & 6,87 & 6,81 & 6,81 & 6,00 &,- 023 & 831 &,- 797 &, $008 * * *$ & ,068 & 877 & -653 & ,114 \\
\hline 15 Implicaciones de las políticas gubernamentales & 6,94 & 6,80 & 6,68 & 5,91 &, 058 & 625 &,- 781 &, $014 * *$ &,- 233 & 612 &,- 973 &, $017 * *$ \\
\hline
\end{tabular}

${ }^{a}$ Los nombres de las competencias han sido obtenidos de http://www.kent.ac.uk/careers/sk/skillsmenu.htm . *, **, *** muestran si la diferencia es significativa al $10 \%, 5 \%$ y $1 \%$ respectivamente. Fuente: elaboración propia.

Octubre 4-6, 2017, Zaragoza, ESPAÑA

IV Congreso Internacional sobre Aprendizaje, Innovación y Competitividad (CINAIC 2017) 